\title{
A SYMBOL SET FOR DIAGRAMMING NETWORKS
}

\author{
Vladan Jovanovic Georgia Southern University vladan@georgiasouthern.edu \\ Eric Stone Georgia Southern University estone4@georgiasouthern.edu
}

\begin{abstract}
This paper presents academic requirements for a standardized symbol set to be used for network diagramming. The paper also illustrates use of a working prototype of the proposed language SANDS (Standardized Network Diagramming Symbols) implemented as a free stencil in Visio.
\end{abstract}

Keywords: Network Diagramming Symbols, Logical Networks, Network Security, Visual Language

\section{INTRODUCTION}

Problems addressed by this paper are related to formulation of academic requirements for a network diagramming language i.e. concepts to address, expressive capabilities, intended use, and selection (evolution) of a simple set of symbols for standardization and similar. The paper presents preliminary results of one year of work on the evolution of a SANDS language in the context of its use in teaching Networking and Security classes.

Text is organized in two main sections:

a) Requirements section, that presents collected and organized requirements (obtained in collaboration with diverse faculty teaching 'networking' across the curriculum in the CS, IS, and IT programs),

b) Selected symbols and a proof of concept prototype implementation in the form of a Visio stencil, illustrating recommended use by examples (more elaborate ones will be presented and posted on the web).

\section{REQUIREMENTS}

As academics teaching networking, information security (assurance) and storage oriented courses we are aware of the problem faced by students and professionals alike, namely the absence of a standardized set of symbols for representing networks. Different texts use variations of device pictures, a few more or less recognizable symbols from systems diagrams and as needed additionally invented symbols rather freely and students have to cope with this at least at three levels: a) separation of concepts from products (brands), b) recognition of concepts when different 'local' symbols are used and c) drawing the diagrams by hand or using a drawing software tool like Visio. Vendors, particularly Cisco, are evolving their own set of symbols in apparently ad hoc manner, and with great deal of attention to marketing of their products. True, the networking technology is rapidly evolving and is intrinsically complex, but that makes a need to systematically address logical network diagrams academically urgent. A fairly typical example, the Figure-1, of icons used in [1], makes obvious why no one will attempt to draw current network diagrams by freehand on a chalkboard, obviously everyone can attest to inappropriateness for academic use of such complex drawings. Network designers and administrators also face this problem when designing and discussing evolution of their network infrastructures. Let us look at the symbols in [1] with some scrutiny a little further to expose imperfections and obvious inconsistencies such as: four different icons are used for switches, two differently looking workstations respectively labeled as SUN and a Cisco Works, a picture of a laptop used as an icon for a laptop, etc. Everyone can review Figure 1 for more examples of arbitrary choices of symbols. A relatively low quality image on Figure 1 is deliberately chosen to illustrate what a student may see on a black and white copy in their handouts where suggested 3D perspective-shading is simply taking space and blurring the image of every icon; the actual reproduced copy in scanned directly from [1] and portrays icons in the same size as used in most texts.

\section{$<$ insert Figure-1 here $>$}

Everyone seems to be too busy to think diagramming through and even aware academics seems to be unwilling to even try to establish a prototype for a standard set of symbols for logical network representation. Several obstacles appear objective, for example uncertainty of answers to pertinent questions such as: a) will a student exposed to one standard have difficulties in recognizing legacy diagrams and adjusting to established diagramming practices (by vendors) in industry, b) will authors of networking texts 'buy-into' a 'common' standard and c) how one can expect a 'standard' to take roots? Inertia can be a 
good thing when an existing solution is adequate but we claim that existing situation with practice of network diagramming is not satisfactory. So we decided to empirically collect requirements for a set of logical symbols, a simple language for drawing networks and to separate design of such a language from possibly even harder practical problem of selling it.

Desirable features of the intended visual language for network diagramming, elicited from a number of consulted faculty lead to the following initial list of requirements:

- $\quad$ Symbols should be logical i.e. abstract, not overly representational, more like road signs (for example not a picture of a brick wall in case of a firewall)

- Preferably common and non proprietary symbols well established internationally, for example a communication link and a disk based storage.

- Appropriate for visualizing functional and structural information for multilevel networks (subnets),

- Sharp and clear 2D (minimal number of lines, painted and exposed surfaces and no shading)

- Memorable that is easy to recognize,

- $\quad$ Crisp in black and white copies, and

- $\quad$ Easy to draw by hand and by a common tools like Visio (stencil).

Specifically symbols should address logical network diagrams at all layers and in various domains such as:

o Traditional (analog) networks

o Data networks

o LAN/CAN/MAN/WAN

o Internet/Intranet

o Wireless networks, incl. Satellite ones

o Optical networks

o Storage networks, SAN, NAS, CAS arrays etc.

o Distributed Computing networks

o Supercomputing architecture,

o Security Assessment (with additional overlays showing DMZ, infestation/recovery paths, possibly honey pots etc. etc.).

Components expected should be the one frequently used in modern networks, for example a minimal selection from a larger list such as:

o Links- cables etc., buses, hubs, phones, servers (computers), external memory (disk), internal memory and cache memory, repeaters, modems, Host Bus Adapters, multiplexers, CSU/CDU, transceivers, access points, printers, scanners, cameras, satellites, antennae, ports, connectors, adapters/interfaces, taps, concentrators, sensors, amplifiers, etc. bridges, routers, switches, gateways, encryption devices, IDS, firewalls, storage arrays, and multifunctional devices or enclosures for honey-pots, etc. many of which are prominent at multiple layers.

The key issue to start with is which symbols to use, and an answer to this question is to be based on explicit set of criteria and an academic assessment of established practices, with an expectations that most symbols can be reused as is or abstracted into minimal and consistent set. As network is formally a graph whose arcs are communication lines (various kinds of channels including implied as in case of a broadcast) and whose nodes are functional devices transforming content and/or regulating communication traffic, various symbols are needed for nodes and links.

To rigorously define a formal language is a task clearly to be done; we postponed formalization for some later time as our selection will be initially based on common usage to agree with intuition of general users. Furthermore, we intent to explore possibility of involving IEEE or similar standardization bodies in considering consensus based framework for official standard development and adoption once a solid requirements become acceptable for a wider community of stakeholders (that is beyond our primary focus on academics and students).

Collecting of requirements outlined above was performed during two semesters in consultation with a number of instructors involved in teaching networking related coursework in the IS, CS and IT departments and a number of very enthusiastic students. A professional conference like IACIS may be a good venue to vet those requirements and evaluate an initial prototype as well as provide support and momentum for eventual improvement and most of all early adoption.

Decision to focus our attention exclusively on logical representation opened up a need to consider annotations for the symbols thus providing for media and performance information to be added to a diagram (still avoiding brand names etc. as appropriate). We think of the selection of rules for combining and connecting symbols and/or adding 
annotations to symbols as a form of syntax rules or a grammar of a symbolic language whose terminal components are symbols - this is also the next big question that need an answer if we are to define a reasonably general, complete and consistent standard for logical diagramming of networks as systems.

\section{INITIAL RECOMMENDATIONS}

A set of symbols (following consultations with diverse faculty) for a proof of concept prototype is:

1. Network as a whole (cloud)

2. Communication line (link/bus)

3. Server

4. Client

5. Storage (Disk)

6. Switch

7. Router

8. Hub

9. Bridge

10. Firewall

11. IDS

12. Antennae

13. Satellite

14. WAP

15. Modem (or more generally adapter)

16. Repeater

17. Encrypting Device (Enigma)

18. Honey pot- represented as a separate node symbol i.e. functional device

19. Enclosure (packages for combining any number of functional blocks)

20. Overlay- with dotted lines (DMZ, etc.)

While brand information is unnecessary at logical level, useful annotation content can be added and standardized to cover relevant information such as: name, id, media, protocol, and performance characteristics and constraints.

The SANDS had been experimentally tested in several classes and is winning appreciation by students and faculty for important reasons, namely:

a) it is very easy to include in a course (it takes less than 20 minutes including drawing a first example by students).

b) A Visio stencil for SANDS prototype is freely available for all interested parties and is demonstrably easy to use.

c) In addition an number of diagrams, examples illustrating typical usage is also readily available.

At this stage, symbols are not widely recognized, but satisfaction of major requirement, easy of use for a free hand drawing and with a Visio is clearly demonstrated. Icons used in SANDS prototype are shown in Figure 2.

\section{<insert Figures 2 and 3 here>}

The images are clearly more visible and retain that property even when minimized as in the print screen example in Figure 3.

\section{CONCLUSIONS}

Work ahead is a combination of refinement (for example: Virtualization, Storage Network extensions etc.), a broad dissemination effort, and three research challenges:

a) Formal Language Definition

b) Evaluation of effectiveness and

c) Adding analytical capabilities.

In terms of refining and evolving the SANDS in typical academic environments, we are currently testing SANDS for possible extensions in depicting network storage technology and visualization. Furthermore comments from trial use and preliminary validation based on coverage in a diverse sample of professional sources [2, 3, 4, 5, 6 and 7] are collected while further experiments and refinements are in the planning stage. We are of the opinion that a need for a standard set of symbols like the SANDS is real and urgent and that a coordinated effort of a wider academic community is warranted, so the main purpose of presenting our early prototype is a proof of concept and a call for action.

\section{REFERENCES}

1. W. Odom, "CCNA ICDN- Exam Certification Guide”, Cisco Press 2004,

2. G. Holden, "Guide to Firewalls and Network Security- Intrusion Detection and VPNs”, Course Technology 2004,

3. M. Chiampa, "Security+ Guide to Network Security Fundamentals”, $2^{\text {nd }}$ edition, Course technology 2005,M. Osborne, "How to Cheat at Managing Information Security”, Syngress 2006,

4. A. Chiarella, "CompTIA Network+ Self-Study Guide”, $2^{\text {nd }}$ edition, Thomson 2006,

5. J. Long “Storage Networking Protocols” Cisco Press 2006,

6. R. Froom, "Building Cisco Multilayeer Switched networks (BCMSN) $4^{\text {th }}$ edition, Cisco Press 2007,

7. B. Forouzan, "Cryptography and Network Security”, McGraw Hill 2008. 


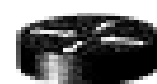

Fruter

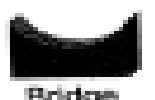

Bridas

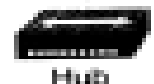

Hub
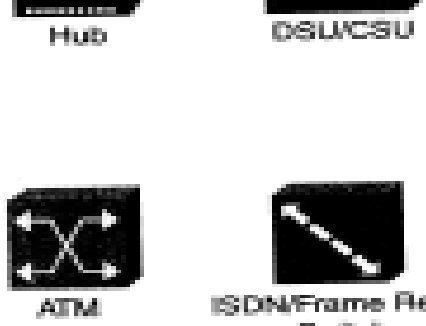

Swith
SDNFrame Pow Switoh

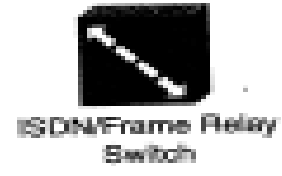

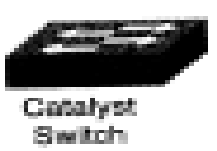

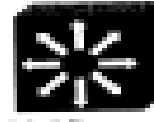

tutisy
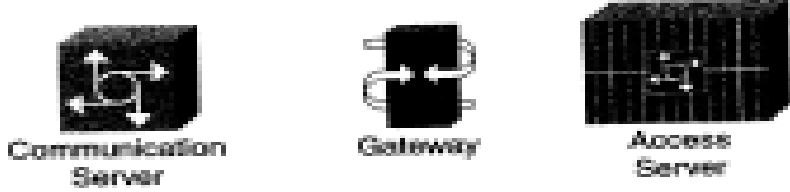

Berut
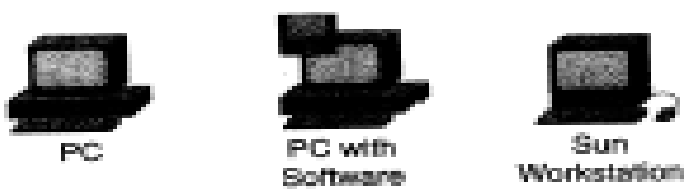

Worktotat

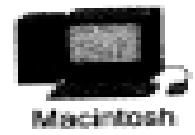

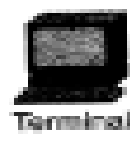

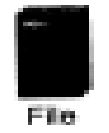

Earnar

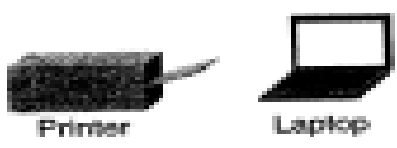

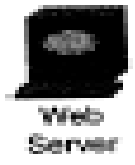

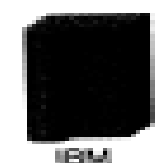

Mintrame
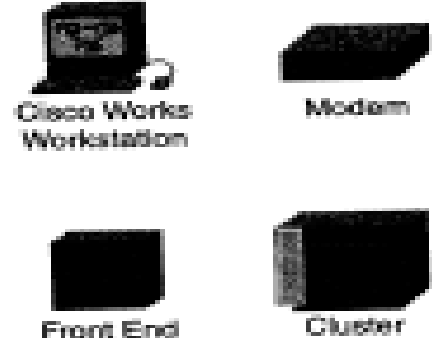

Fich: Ens

Froosesar

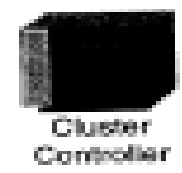

Figure 1. Icons used in [1]

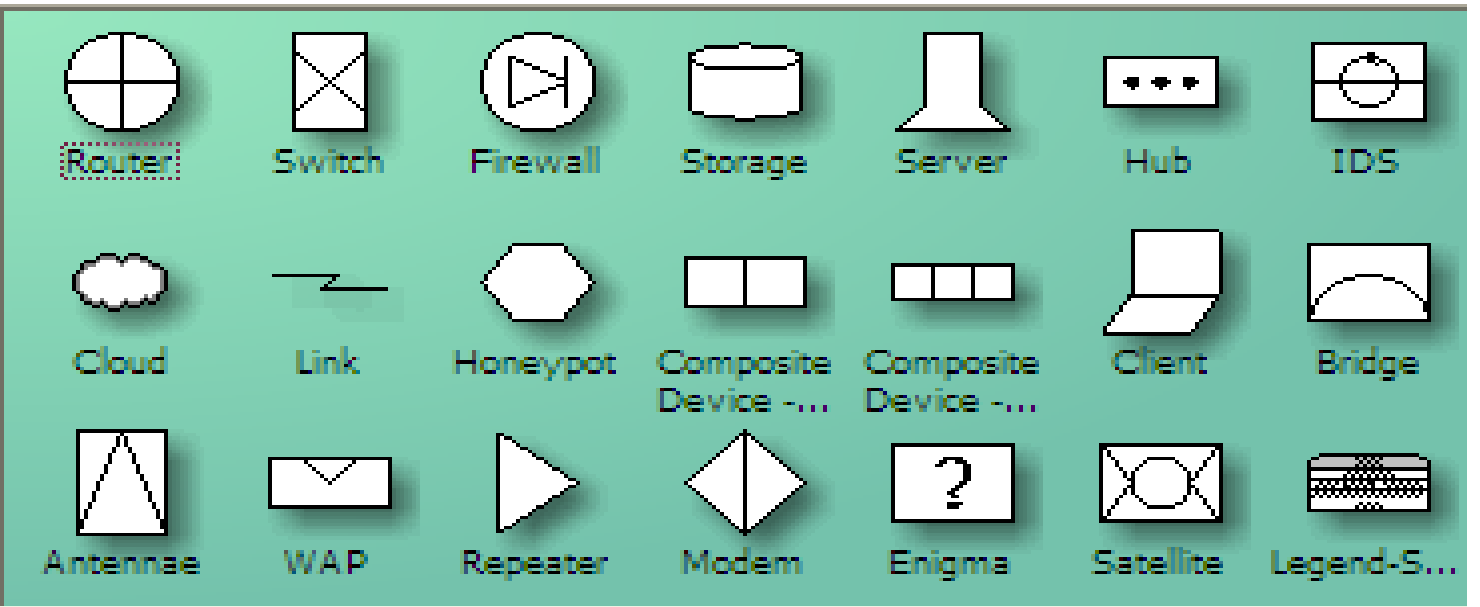

Figure2. Icons used in SANDS prototype version 0.1 


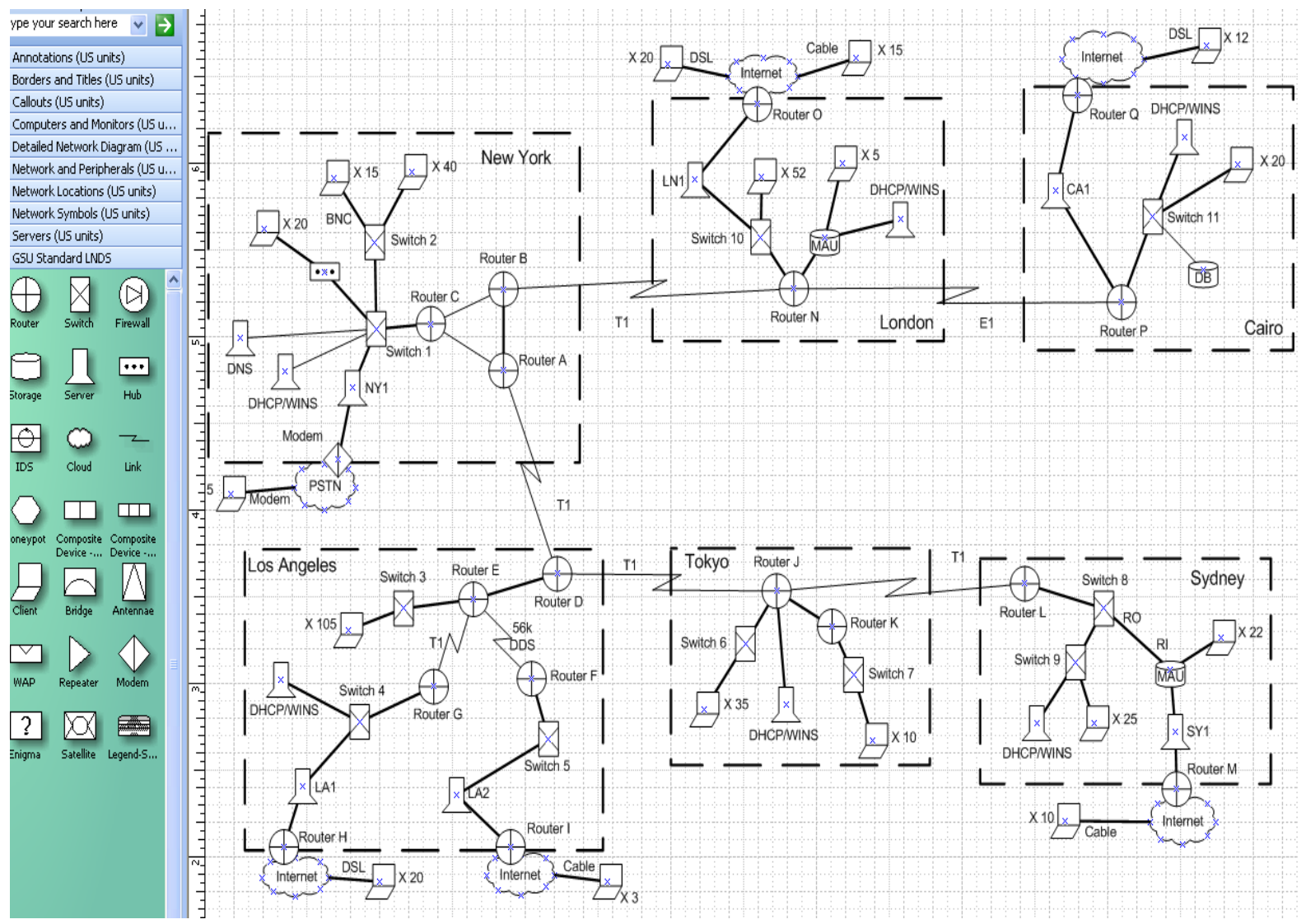

Figure 2. An example of SANDS use by students during preliminary validation of the prototype version 0.1 